\title{
Propranolol therapy for infantile hemangioma and proposed protocol for initiation of therapy
}

Volume 2 Issue I - 2015

\section{Editorial}

Infantile hemangiomas (IHs) are the most common vascular tumors of infancy, found in approximately $5-10 \%$ of all newborns and infants up to lyear. IHs are self limited and in most of the cases no intervention is needed. Approximately $10 \%$ of all $\mathrm{IHs}$ require medical intervention, include those involving areas such as: face (periorbital, perinasal, perioral) or sites at high risk for ulceration (skin folds, anogenital area), causing airway obstruction, dysfunction, hemorrhage or disfigurement. ${ }^{1}$

In 2008 Leaute-Labreze et al., ${ }^{2}$ reported for the first time about propranolol treatment for infantile hemangioma. Since then propranolol has become a first line treatment for IHs, replacing older treatments like systemic steroids, vincristine, cyclophosphamide, interferon alfa-2a, pulsed dye laser and surgery. Propranolol is being used for cardiovascular indications such as: angina pectoris, hypertension, tachyarrhythmias, myocardial infarction, and for other non-cardiogenic indications including essential tremor, migraine, cluster headaches and thyrotoxicosis. Propranolol blocks the action of epinephrine and norepinephrine on both $\beta_{1}$ - and $\beta_{2}$-adrenergic receptors since it is non-selective beta blocker. The mechanism of action of propranolol on IHs is not fully understood, and thought to involve three potential effects: vasoconstriction, inhibition of angiogenesis and induction of apoptosis of the endothelial cells. ${ }^{3}$

Overall, propranolol has good safety profile. It has been used in the pediatric population for years to manage hyperthyroidism, supraventricular tachyarrythmias and other types of arrhythmias, and in the last years has become the first-line therapy for IHs. Adverse effects of propranolol in treatment of IHs as reported in consensus conference and later published were: sleep disturbances (3.7\%), asymptomatic or unspecified hypotension (2.8\%), somnolence $(2.2 \%)$, cool or mottled extremities $(1.7 \%)$, pulmonary symptoms including wheezing $(1.4 \%)$, asymptomatic or unspecified bradycardia $(0.9 \%)$, hypoglycemia $(0.9 \%)$, gastroesophageal reflux or gastrointestinal upset $(0.7 \%)$, symptomatic hypotension $(0.3 \%)$, and symptomatic bradycardia $(0.1 \%)^{4}$. The most worrisome adverse effect is hypoglycemia, although it can be minimized with proper administration of the drug following feeding and parents' education. Other worrisome side effects are those related to the cardiovascular system (hypotension, bradycardia), but these were found to be at their peak 2hours following administration, and usually after the first dose, ${ }^{5}$ which is manageable if done under medical supervision.

Propranolol approved recently by the FDA for the treatment of IHs in dosage of up to $3 \mathrm{mg} / \mathrm{kg} / \mathrm{day}$, but to date there is no consensus guidelines regarding the dosage or the duration of treatment of the propranolol for IHs. Our suggested protocol which is based on the literature and our experience regarding initiation of propranolol therapy is the following: propranolol should be initiated at a dose of $0.5 \mathrm{mg} / \mathrm{kg} /$ day divided in to 3 equal doses. In the following 72 hours

\author{
Amir Horev,' Alex Zvulunov²,3 \\ 'Pediatric Dermatology Service, Soroka University Medical \\ Center, Israel \\ ${ }^{2}$ Pediatric Dermatology Unit, Schneider Children's Medical \\ Center of Israel, Israel \\ ${ }^{3}$ Medical School for International Health, Ben-Gurion University \\ of the Negev, Israel
}

Correspondence: Amir Horev, Pediatric dermatology service, Soroka university medical center, Yitzhak Rager Ave, P.O Box I5I, Beer-Sheva, 84 I 0 I, Israel, Tel (972-8) 624-4667, Fax (972-8) 640-3916, Email amirhor@clalit.org.il

Received: December 05, 2014 | Published: January 02, 2015

it should gradually increase to a target dose of $2 \mathrm{mg} / \mathrm{kg} / \mathrm{day}$. We recommend that the initiation of therapy would be under supervision either in the department of pediatrics or the day hospitalization department. Blood pressure, pulse rate and blood glucose level should be monitored. Treatment is best terminated at age 12-15months, when the proliferative stage of the IHs ends. ${ }^{6}$ Parents should be guided to feed the infant every 4-6hours to avoid possible hypoglycemia.

In summary, propranolol is considered today to be the first line treatment for IHs that needs medical care due to its high efficacy and fewer side effects comparing to steroids, vincristine, cyclophosphamide and others. We suggested a protocol for safe initiation of propranolol therapy for IHs.

\section{Acknowledgments}

None.

\section{Conflicts of interest}

Author declares there are no conflicts of interest.

\section{Funding}

None.

\section{References}

1. Xiao Q, Li Q, Zhang B, et al. Propranolol therapy of infantile hemangiomas: efficacy, adverse effects, and recurrence. Pediatr Surg Int. 2013; 29(6):575-581.

2. Leaute-Labreze C, Dumas de la Roque E, Hubiche T, et al. Propranolol for severe hemangiomas of infancy. $N$ Engl $\mathrm{J} \mathrm{Med}$. 2008;358(24):2649-2651. 
3. Storch $\mathrm{CH}$, Hoeger PH. Propranolol for infantile haemangiomas: insights into the molecular mechanisms of action. $\mathrm{Br} J$ Dermatol. 2010;163(2):269-274.

4. Drolet BA, Frommelt PC, Chamlin SL, et al. Initiation and use of propranolol for infantile hemangioma: report of a consensus conference. Pediatrics. 2013;131(1):128-140.
5. Patel NJ, Bauman NM. How should propranolol be initiated for infantile hemangiomas: inpatient versus outpatient? Laryngoscope. 2014;124(6):1279-1281.

6. Sagi L, Zvulunov A, Lapidoth M, et al. Efficacy and safety of propranolol for the treatment of infantile hemangioma: a presentation of ninety-nine cases. Dermatology. 2014;228(2):136-144. 